\title{
Reporting diagnostic accuracy studies: where are we now?
}

\section{"...readers need to know whether the study they are reading is applicable to the situation they are in."}

Reporting guidelines are booming. A quick look at the EQUATOR website - the website for research reporting guidelines - revealed over 250 different guidelines, varying from the well-known CONSORT statement for reporting of randomized controlled trials and PRISMA for reporting of systematic reviews to the lesser known ones like TRIPOD for prediction models and CARE for case reports [1]. And after the development of reporting guidelines for full text articles, reporting guidelines for abstracts are now emerging $[2,3]$.

Regardless of the number of existing guidelines, the actual reporting of the items listed in those guideline lays far behind. Several research projects have investigated the adherence to reporting guidelines in different areas of healthcare and they all reveal the same trend: although reporting may be improving in some areas, there is still much that remains unreported. This is not very different for diagnostic accuracy studies. We analyzed 112 diagnostic accuracy studies published in high-impact journals in 2012 and found that on average these studies reported only 15 items out of the complete list of 25. Although this was a significant improvement in comparison to similar analyses done in 2000 (11.9 out of 25) and 2004 (13.6 out of 25), crucial information remains unreported [4]. Even though the included journals all but one mentioned the use of STARD in their guidelines for authors.

Apparently, researchers are either not aware of the existence of these reporting guidelines, or they do not consider using them. How is this possible? What are the drivers for an author to adhere to these guidelines and how can we increase adherence?
Until now, efforts were mainly put into the development of the tools and it seemed as if the developers expected external forces, such as journal editors and peer reviewers, to force adherence upon authors. However, not all journals do include STARD in their authors guidelines and even if they do, from our previously mentioned analyses it may be concluded that not all journals indeed check the adherence to STARD. Furthermore, not all peer reviewers may be aware of the need of complete reporting, or they may find it cumbersome to check whether indeed all items of STARD have been reported. Although one may find that journals and peer reviewers should have the moral obligation to ask for complete reporting, these incentives have proven not to work sufficiently.

Although these strategies may be improved, for example by using electronic pop-ups as soon as someone submits a diagnostic accuracy paper, these external incentives only act after most of the work has already been done. Furthermore, research done in other fields shows that people need both external incentives and intrinsic motivation to change their behavior [5,6]. Intrinsic motivation to use reporting guidelines as a useful tool throughout the research project should therefore be promoted.

Intrinsic motivation for authors to adhere to reporting guidelines may come from the understanding of why complete reporting is important. There are several reasons for this and I will discuss a few. First of all, readers of these studies need to know whether the study they are reading has a potential for bias. If there is potential for bias, the study is not immediately useless. Readers must be enabled to estimate the extent of bias and the direction

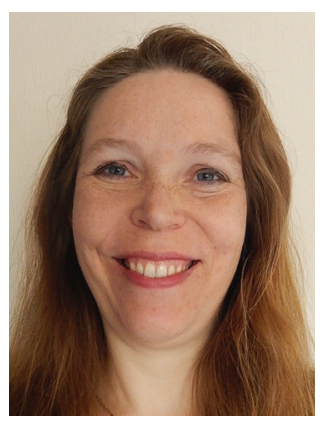

Mariska MG Leeflang Clinical Epidemiology \& Biostatistics \& Bioinformatics; Academic Medical Center, University of Amsterdam, 1012 WX Amsterdam, Netherlands m.m.leeflang@amc.uva.nl 
ing reporting guidelines in courses on good scientific behavior or on the ethics of research may be helpful as well. Healthcare professionals base their decisions upon available evidence. Research reports may directly affect patients and not reporting patient characteristics or study limitations may lead to the wrong decision, thus harming patients.

\section{6 \\ “...a reporting checklist may be useful help while writing the report, or even in the design of the study. Authors should see these reporting guidelines as a helpful tool, rather than a burden.}

Finally, the developers of these reporting guidelines will have to increase awareness among researchers, and to make the checklists easier to access and easier to understand. People need to be aware of the checklists to be able to use them. If finding them takes too long and completing the checklist is too complicated, people will refrain from using them as well. Over the past months, STARD the reporting guidelines for diagnostic accuracy studies, have been revised into STARD 2015. STARD 2015 aims to be simpler for authors to adhere to and more in line with other reporting guidelines. But the most important feature of STARD 2015 is the simultaneous launch of online material and

\section{References}

1 Equator network. www.equator-network.org/

2 Hopewell S, Clarke M, Moher D et al. the CONSORT Group. CONSORT for reporting randomised trials in journal and conference abstracts. Lancet 371(9609), 281-283 (2008).

3 Beller EM, Glasziou PP, Altman DG et al. PRISMA for Abstracts Group. PRISMA for Abstracts: Reporting Systematic Reviews in Journal and Conference Abstracts. PLoS Med. 10(4), e1001419 (2013).

4 Korevaar DA, Wang J, van Enst WA et al. Reporting diagnostic accuracy studies: some improvements after 10 years of STARD. Radiology 274(3), 781-789 (2015).

5 Staunton L, Gellert P, Knittle K, Sniehotta FF. Perceived control and intrinsic vs. extrinsic motivation for oral examples to help authors to understand the requested items. The developers of this checklist no longer expect the world to pick up the tool and use it, but they put effort in the improvement and dissemination of the tool itself [9].

In summary, the reporting of diagnostic test accuracy studies has slightly improved over the years, which may be due to the introduction of STARD. Still, much remains unreported. To improve this, we need to draw a more positive light on the reporting guidelines and enhance the internal motivation of authors to adhere to these guidelines. But, dear authors, the next step is with you: future diagnostic accuracy studies, will be guided by the STARD guidelines from the start. Please check the EQUATOR website for STARD and other reporting guidelines: www.equator-network.org

\section{Financial \& competing interests disclosure}

The author has no relevant affiliations or financial involvement with any organization or entity with a financial interest in or financial conflict with the subject matter or materials discussed in the manuscript. This includes employment, consultancies, honoraria, stock ownership or options, expert testimony, grants or patents received or pending, or royalties.

No writing assistance was utilized in the production of this manuscript.

self-care: a full factorial experimental test of theory-based persuasive messages. Ann. Behav. Med. 49(2), 258-268 (2015).

6 Ten Cate TJ, Kusurkar RA, Williams GC. How selfdetermination theory can assist our understanding of the teaching and learning processes in medical education. AMEE guide No. 59. Med. Teach. 33(12), 961-973 (2011).

7 Mulherin SA, Miller WC. Spectrum bias or spectrum effect? Subgroup variation in diagnostic test evaluation. Ann. Intern. Med. 137(7), 598-602 (2002).

8 Ochodo EA, de Haan MC, Reitsma JB, Hooft L, Bossuyt PM, Leeflang MM. Overinterpretation and misreporting of diagnostic accuracy studies: evidence of "spin". Radiology 267(2), 581-588 (2013).

9 Equator network. www.equator-network.org/2015/03/31/10-years-of-stard/ 